\title{
Testing the quark cluster model in nucleon-nucleon scattering
}

\author{
M. Lacombe, ${ }^{1}$ B. Loiseau, ${ }^{1}$ R. Vinh Mau, ${ }^{1}$ P. Demetriou, ${ }^{2}$ J. P. B. C. de Melo, ${ }^{3}$ and C. Semay ${ }^{4}$ \\ ${ }^{1}$ Laboratoire de Physique Nucléaire et des Hautes Energies, LPTPE, Université Pierre et Marie Curie, 4 Place Jussieu, \\ F-75252 Paris CEDEX 05, France \\ ${ }^{2}$ Institute of Nuclear Physics, NCSR “Demokritos," GR-15310 Athens, Greece \\ ${ }^{3}$ Instituto de Fisica Teorica, IFT, (UNESP) rua Pamplona, 145, Bela Vista, Cep 01405-900 Sao Paulo, Brazil \\ ${ }^{4}$ Université de Mons-Hainaut, Faculté des Sciences, 20 Place du Parc, B-7000 Mons, Belgium
}

(Received 4 July 2001; published 15 February 2002)

\begin{abstract}
The description of the short-range part of the nucleon-nucleon forces in terms of quark degrees of freedom is tested against experimental observables. We consider, for this purpose, a model where the short-range part of the forces is given by the quark cluster model and the long- and medium-range forces by well established meson exchanges. The investigation is performed using different quark cluster models coming from different sets of quark-quark interactions. The predictions of this model are compared not only with the phase shifts but also directly with the experimental observables. Agreement with the existing $p p$ and $n p$ world set of data is poor. This suggests that the current description of the nucleon-nucleon interaction, at short distances, in the framework of the nonrelativistic quark models, is at present only qualitative.
\end{abstract}

DOI: 10.1103/PhysRevC.65.034004

PACS number(s): 21.30.-x, 13.75.Cs

\section{INTRODUCTION}

It has been clearly shown, in many investigations (see Refs. [1-3] and references cited therein), that the rich body of highly accurate data on nucleon-nucleon $(N N)$ scattering, accumulated during the last three decades, can be very successfully described by theoretical models based on hadronic degrees of freedom. The main drawback met in these works is that, for a precise fit to the data, either the concepts of boson exchange are illegimately extended to the very short internucleon distances [4], or a purely phenomenological model is explicitly adopted for these short distances [5]. On the other hand, the nucleon is a compound system of subhadronic particles and the quark degrees of freedom are expected to play a role in the short-range (SR) part where the two nucleons can overlap significantly.

In the past, several attempts [6-9] have been devoted to the derivation of a $N N$ potential from the quark degrees of freedom, namely, in the framework of the so-called quark cluster model (QCM). This model extends the nonrelativistic quark model, which is very successful in accounting for the baryon static properties, to the description of the interaction between two clusters of three quarks. One of the outcomes of these works is that the dominant potential obtained is repulsive for all internucleon distances. This property, of course, is desirable for short distances but the lack of attraction in the medium range is very troublesome. Subsequent works $[10,11]$ remedy this defect by adding to the QCM some meson exchanges. However, these meson exchanges are adjusted to give a best fit of the $N N$ phase shifts. The result is that the obtained medium-range $N N$ forces are then too strongly attractive to be realistic [12]. This feature was confirmed very recently [13] in a new analysis of the high partial waves reported in Ref. [11]. Introducing quark delocalization and color screening to the QCM [14] can furnish some attraction, but this attraction lies in a region far outside that observed.
In contrast, the long- and medium-range (LR and MR) nucleon-nucleon forces provided by meson exchanges are nowadays well established and well tested against the lowenergy data, and we believe that they must be taken as fixed. In this work, we adopt this viewpoint, and investigate a model in which these LR and MR forces are supplemented with the SR forces derived from the quark cluster models. We believe that this procedure brings a better insight into the role played by quark degrees of freedom in the nucleonnucleon interaction, and provides a more meaningful test of the QCM. In a previous work [12], such an analysis has been performed only with the QCM reported in Ref. [11], and for the $p p$ scattering. Here, we extend it to other QCM derived from different quark-quark interactions, and we also compare the predictions with the updated set of data, for both the $p p$ and $n p$ scattering, up to the laboratory kinetic energy $T_{l a b}$ $=350 \mathrm{MeV}$.

Our model as well as the input ingredients are presented in Sec. II. The results are reported and discussed in Sec. III.

\section{THE MODEL}

The model that fulfils the previous requirements, amounts to the Schrödinger equation

TABLE I. Quark-quark interaction input parameters in different QCM.

\begin{tabular}{lccccc}
\hline \hline \multicolumn{1}{c}{ QCM } & $\begin{array}{c}m_{q} \\
(\mathrm{MeV})\end{array}$ & $\begin{array}{c}b_{q} \\
(\mathrm{fm})\end{array}$ & $\alpha_{s}$ & $\begin{array}{c}a_{1} \\
\left(\mathrm{MeV} \mathrm{fm}^{-1}\right)\end{array}$ & $\begin{array}{c}a_{2} \\
\left(\mathrm{MeV} \mathrm{fm}^{-2}\right)\end{array}$ \\
\hline Takeuchi [11] & 313.0 & 0.62 & 1.657 & 43.84 & \\
Yamauchi [15] & 313.0 & 0.50 & 0.360 & & 67.27 \\
Oka [6] & 300.0 & 0.60 & 1.392 & 141.20 & \\
Faessler [7] & 355.0 & 0.475 & 0.970 & 61.60 & \\
\hline \hline
\end{tabular}


TABLE II. Harvey [16] input parameters.

$$
\begin{array}{cc}
-\frac{1}{m_{N}} \boldsymbol{\nabla}^{2} \psi(\mathbf{r})+V_{P}(\mathbf{r}, E)[1-f(r)] \psi(\mathbf{r}) & V_{i j}^{r e s}=V_{i j}^{O G E}=\frac{\alpha_{s}}{4} \boldsymbol{\lambda}_{i} \cdot \boldsymbol{\lambda}_{j}\left[\frac{1}{r_{i j}}-\frac{\pi}{m_{i}^{2}}\left(1+\frac{2}{3} \boldsymbol{\sigma}_{i} \cdot \boldsymbol{\sigma}_{j}\right) \delta\left(\mathbf{r}_{i j}\right)\right. \\
+\int d \mathbf{r}^{\prime} V_{Q C M}\left(\mathbf{r}, \mathbf{r}^{\prime}\right) \sqrt{f(r) f\left(r^{\prime}\right)} \psi\left(\mathbf{r}^{\prime}\right)=E \psi(\mathbf{r}), & \left.-\frac{1}{m_{i}^{2} r_{i j}^{3}}\left(3 \boldsymbol{\sigma}_{i} \cdot \mathbf{r}_{i j} \boldsymbol{\sigma}_{j} \cdot \mathbf{r}_{i j}-\boldsymbol{\sigma}_{i} \cdot \boldsymbol{\sigma}_{j}\right)-\frac{3}{2 m_{i}^{2} r_{i j}^{3}} \mathbf{L} \cdot \mathbf{S}\right],
\end{array}
$$

\begin{tabular}{cccccccc}
\hline \hline $\begin{array}{c}K \\
\left(\mathrm{MeV} \mathrm{fm}^{-3}\right)\end{array}$ & $\begin{array}{c}m_{q} \\
(\mathrm{MeV})\end{array}$ & $\alpha_{s}=-4 m_{q}^{2} K / \pi$ & $\begin{array}{c}b_{q} \\
(\mathrm{fm})\end{array}$ & $\begin{array}{c}A \\
(\mathrm{MeV})\end{array}$ & $\begin{array}{c}B \\
\left(\mathrm{MeV} \mathrm{fm}^{-2}\right)\end{array}$ & $\begin{array}{c}C \\
(\mathrm{fm})\end{array}$ & $\begin{array}{c}\Lambda \\
(\mathrm{MeV})\end{array}$ \\
\hline-227.77 & 355 & 4.757 & 0.8 & 952.5 & -3.125 & -119.95 & 0.8 \\
\hline \hline
\end{tabular}

where $V_{P}(\mathbf{r}, E)$ is the theoretical LR + MR part of the Paris potential, given by the one-pion-exchange, the uncorrelated and correlated two-pion exchange contributions [5]. This potential is local but energy dependent. $V_{Q C M}\left(\mathbf{r}, \mathbf{r}^{\prime}\right)$ is the QCM nucleon-nucleon potential due to the quark confinement plus the one-gluon exchange contributions. It contains a local and a nonlocal part but is energy independent. $f(r)$ $=\left[1+\left(r / R_{c}\right)^{p}\right]^{-1}$ is a cutoff function designed to make a clear separation between the SR and LR+MR parts of the interaction.

For completeness, let us sketch briefly the derivation of the QCM potential $V_{Q C M}\left(\mathbf{r}, \mathbf{r}^{\prime}\right)$ in terms of the inputs, namely, the quark-quark interactions. The Hamiltonian considered for the six-quark system is

$$
H=\sum_{i=1}^{6} \frac{p_{i}^{2}}{2 m_{i}}-T+\sum_{i<j} V_{i j}
$$

$T$ is the kinetic energy for the c.m. motion. The quark-quark interaction $V_{i j}$ consists of the confinement term $V_{i j}^{\text {conf }}$ which is supposed to account for the nonperturbative effects of QCD and of the residual interaction $V_{i j}^{r e s}$

$$
V_{i j}=V_{i j}^{\text {conf }}+V_{i j}^{r e s}
$$

Usually the confinement potential is taken to be linear or quadratic

$$
V_{i j}^{c o n f}=-\left(\boldsymbol{\lambda}_{i} \cdot \boldsymbol{\lambda}_{j}\right) a_{n} r_{i j}^{n} \quad(n=1 \quad \text { or } 2)
$$

and the residual interaction is given by the one-gluon exchange potential

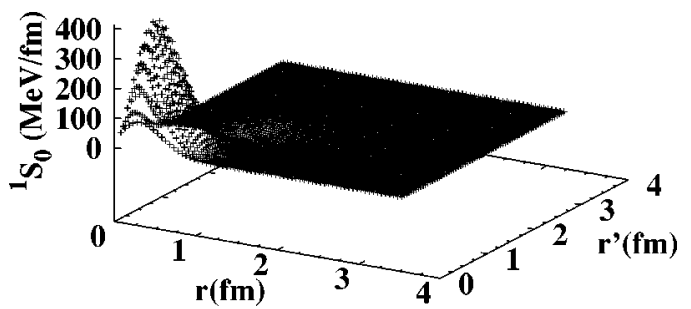

FIG. 1. Nonlocal ${ }^{1} S_{0}$ partial wave projection of $r r^{\prime} V_{Q C M}\left(\mathbf{r}, \mathbf{r}^{\prime}\right)$, for the parameter set of Ref. [11]. where $\boldsymbol{\lambda}_{i}$ are the color matrices.

In the quark cluster model, the wave function for a sixquark system is assumed to be of the form

$$
\Psi_{\beta}\left(\boldsymbol{\xi}_{a}, \boldsymbol{\xi}_{b}, \mathbf{r}_{a b}\right)=\mathcal{A}\left[\left\{\phi_{a}(123) \phi_{b}(456)\right\}_{\beta} \chi_{\beta}\left(\mathbf{r}_{a b}\right)\right],
$$

where $\phi_{a, b}$ is the wave function of nucleon $a$ or $b$ (cluster of three quarks), $\chi_{\beta}$ the relative wave function, $\mathbf{r}_{a b}=\mathbf{r}_{a}-\mathbf{r}_{b}$ the relative coordinate between the two clusters centered at $\mathbf{r}_{a}$ $=\frac{1}{3}\left(\mathbf{r}_{1}+\mathbf{r}_{2}+\mathbf{r}_{3}\right)$ and $\mathbf{r}_{b}=\frac{1}{3}\left(\mathbf{r}_{4}+\mathbf{r}_{5}+\mathbf{r}_{6}\right), \mathcal{A}$ is the antisymmetrization operator with respect to the quarks and $\beta$ the coupling of $a$ and $b$ into a well-defined flavor and spin state $\beta$.

The Schrödinger equation gives

$\sum_{\beta} \int\left\{\phi_{a}^{*}\left(\boldsymbol{\xi}_{a}\right) \phi_{b}^{*}\left(\boldsymbol{\xi}_{b}\right)\right\}_{\alpha}[H-E] \Psi_{\beta}\left(\boldsymbol{\xi}_{a}, \boldsymbol{\xi}_{b}, \mathbf{r}_{a b}\right) d \boldsymbol{\xi}_{a} d \boldsymbol{\xi}_{b}=0$,

where the internal coordinates of the clusters $a$ and $b$ are now introduced explicitly as

$$
\begin{gathered}
\boldsymbol{\xi}_{a}=\left(\boldsymbol{\xi}_{1}, \boldsymbol{\xi}_{2}\right) \quad \boldsymbol{\xi}_{b}=\left(\boldsymbol{\xi}_{3}, \boldsymbol{\xi}_{4}\right) \\
\boldsymbol{\xi}_{1}=\mathbf{r}_{1}-\mathbf{r}_{2}, \quad \boldsymbol{\xi}_{2}=\mathbf{r}_{3}-\frac{1}{2}\left(\mathbf{r}_{1}+\mathbf{r}_{2}\right) \\
\boldsymbol{\xi}_{3}=\mathbf{r}_{4}-\mathbf{r}_{5}, \quad \boldsymbol{\xi}_{4}=\mathbf{r}_{6}-\frac{1}{2}\left(\mathbf{r}_{4}+\mathbf{r}_{5}\right) .
\end{gathered}
$$

Straightforward manipulations lead to a coupled channel equation, the resonating group method (RGM) equation

$$
\sum_{\beta} \int d \mathbf{r}^{\prime}\left[H_{\alpha \beta}\left(\mathbf{r}, \mathbf{r}^{\prime}\right)-E N_{\alpha \beta}\left(\mathbf{r}, \mathbf{r}^{\prime}\right)\right] \chi_{\beta}\left(\mathbf{r}^{\prime}\right)=0,
$$

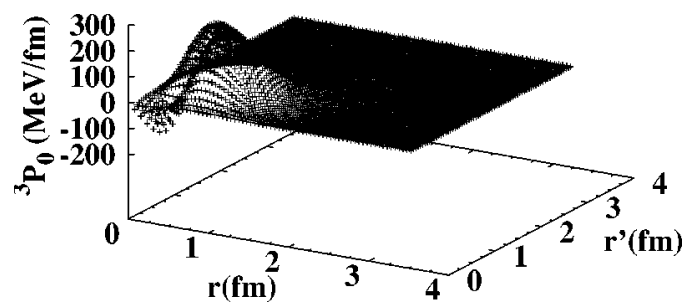

FIG. 2. As in Fig. 1 but for the ${ }^{3} P_{0}$ wave. 


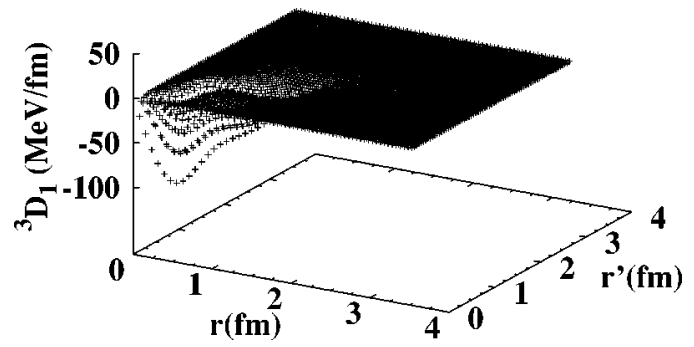

FIG. 3. As in Fig. 1 but for the ${ }^{3} D_{1}$ wave.

where the operators

$$
\begin{aligned}
H\left(\mathbf{r}, \mathbf{r}^{\prime}\right)= & \int \phi_{a}^{+}\left(\boldsymbol{\xi}_{a}\right) \phi_{b}^{+}\left(\boldsymbol{\xi}_{b}\right) \delta\left(\mathbf{r}-\mathbf{r}_{a b}\right) \\
& \times H(1-\mathcal{A}) \phi_{a}\left(\boldsymbol{\xi}_{a}\right) \phi_{b}\left(\boldsymbol{\xi}_{b}\right) \delta\left(\mathbf{r}^{\prime}-\mathbf{r}_{a b}\right) d \boldsymbol{\xi}_{a} d \boldsymbol{\xi}_{b} d \mathbf{r}_{a b} \\
= & H^{d}\left(\mathbf{r}^{\prime}\right) \delta\left(\mathbf{r}^{\prime}-\mathbf{r}\right)-H^{e x}\left(\mathbf{r}, \mathbf{r}^{\prime}\right)
\end{aligned}
$$

and

$$
\begin{aligned}
N\left(\mathbf{r}, \mathbf{r}^{\prime}\right)= & \int \phi_{a}^{+}\left(\boldsymbol{\xi}_{a}\right) \phi_{b}^{+}\left(\boldsymbol{\xi}_{b}\right) \delta\left(\mathbf{r}-\mathbf{r}_{a b}\right) \\
& \times(1-\mathcal{A}) \phi_{a}\left(\boldsymbol{\xi}_{a}\right) \phi_{b}\left(\boldsymbol{\xi}_{b}\right) \delta\left(\mathbf{r}^{\prime}-\mathbf{r}_{a b}\right) d \boldsymbol{\xi}_{a} d \boldsymbol{\xi}_{b} d \mathbf{r}_{a b} \\
= & N^{d}\left(\mathbf{r}^{\prime}\right) \delta\left(\mathbf{r}^{\prime}-\mathbf{r}\right)-N^{e x}\left(\mathbf{r}, \mathbf{r}^{\prime}\right) .
\end{aligned}
$$

The exchange terms $H^{e x}\left(\mathbf{r}, \mathbf{r}^{\prime}\right)$ and $N^{e x}\left(\mathbf{r}, \mathbf{r}^{\prime}\right)$ arise from the antisymmetrization operator

$$
\mathcal{A}=1-\sum_{i=1}^{3} \sum_{j=4}^{6} P_{i j} .
$$

The intrinsic wave functions of the two clusters $\phi_{a}$ and $\phi_{b}$ are usually approximated by the $(O s)^{3}$ harmonic oscilla-

\begin{tabular}{|c|c|c|}
\hline & Isospin 1 & Isospin 0 \\
\hline Models & $\begin{array}{l}\quad R_{c}(\mathrm{fm}) \\
\text { (fitted to }{ }^{1} S_{0} \\
\text { at } 25 \mathrm{MeV} \text { ) }\end{array}$ & $\begin{array}{c}R_{c}(\mathrm{fm}) \\
\text { (fitted to } \\
\text { deuteron binding energy) }\end{array}$ \\
\hline Takeuchi [11] & 0.820 & 0.806 \\
\hline Yamauchi [15] & 0.845 & 0.799 \\
\hline Oka [6] & 0.824 & 0.806 \\
\hline Faessler [7] & 0.841 & 0.806 \\
\hline Harvey [16] & 0.900 & 0.909 \\
\hline
\end{tabular}
tor configurations

$$
\begin{aligned}
\phi\left(\boldsymbol{\xi}_{a}\right)= & \left(\frac{1}{4 \pi b_{q}^{2}}\right)^{3 / 2} \exp \left[-\frac{1}{4 b_{q}^{2}} \xi_{1}^{2}\right]\left(\frac{2}{3 \pi b_{q}^{2}}\right)^{3 / 2} \\
& \times \exp \left[-\frac{1}{3 b_{q}^{2}} \xi_{2}^{2}\right] \Xi^{S, T}(1,2,3),
\end{aligned}
$$

TABLE III. Best values of the cutoff radius $R_{c}$. The models correspond to the different QCM, supplemented with the LR+MR part of the Paris potential.

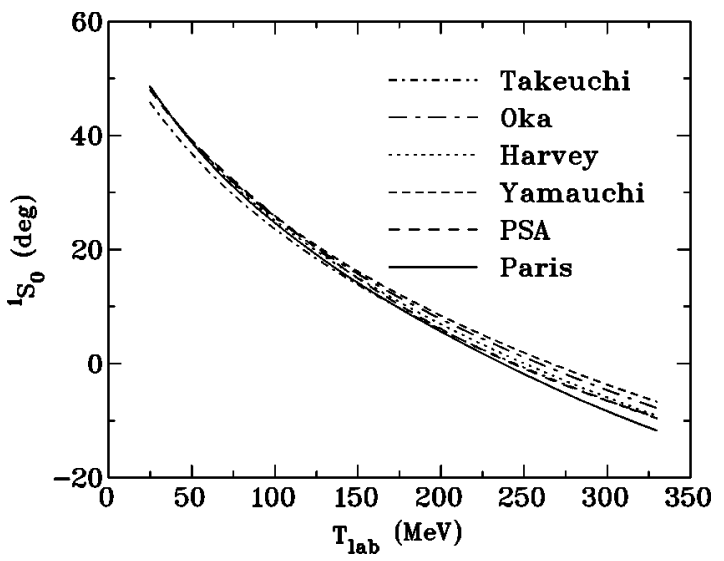

FIG. 4. ${ }^{1} S_{0}$ phase-shifts. The curves correspond to the different QCM, supplemented with the LR+MR part of the Paris potential. PSA corresponds to phase shift analysis [22], and Paris to the results of Ref. [5].

where $b_{q}$ is the oscillator parameter and $\Xi^{S, T}(1,2,3)$ is the spin $(S)$-isospin $(T)$ color wave function.

Introducing the following renormalized wave function:

$$
\tilde{\chi}(\mathbf{r})=\int N^{1 / 2}\left(\mathbf{r}, \mathbf{r}^{\prime}\right) \chi\left(\mathbf{r}^{\prime}\right) d \mathbf{r}^{\prime}
$$

and the corresponding renormalized Hamiltonian kernel

$$
\widetilde{H}\left(\mathbf{r}, \mathbf{r}^{\prime}\right)=\int N^{-1 / 2}\left(\mathbf{r}, \mathbf{r}^{\prime \prime}\right) H\left(\mathbf{r}^{\prime \prime}, \mathbf{r}^{\prime \prime \prime}\right) N^{-1 / 2}\left(\mathbf{r}^{\prime \prime \prime}, \mathbf{r}^{\prime}\right) d \mathbf{r}^{\prime \prime} d \mathbf{r}^{\prime \prime \prime},
$$

one obtains

$$
\int d \mathbf{r}^{\prime} \tilde{H}\left(\mathbf{r}, \mathbf{r}^{\prime}\right) \tilde{\chi}\left(\mathbf{r}^{\prime}\right)=E \tilde{\chi}(\mathbf{r}) .
$$

Identifying this equation with an ordinary Schrödinger-type equation provides the expression of $V_{Q C M}\left(\mathbf{r}, \mathbf{r}^{\prime}\right)$ in terms of the kernels $H\left(\mathbf{r}, \mathbf{r}^{\prime}\right)$ and $N\left(\mathbf{r}, \mathbf{r}^{\prime}\right)$. The full fledged details of these calculations can be found, for example, in Ref. [15].

As for the input parameters of the quark-quark interaction, namely, the quark masses $m_{i}$, the confining strengths

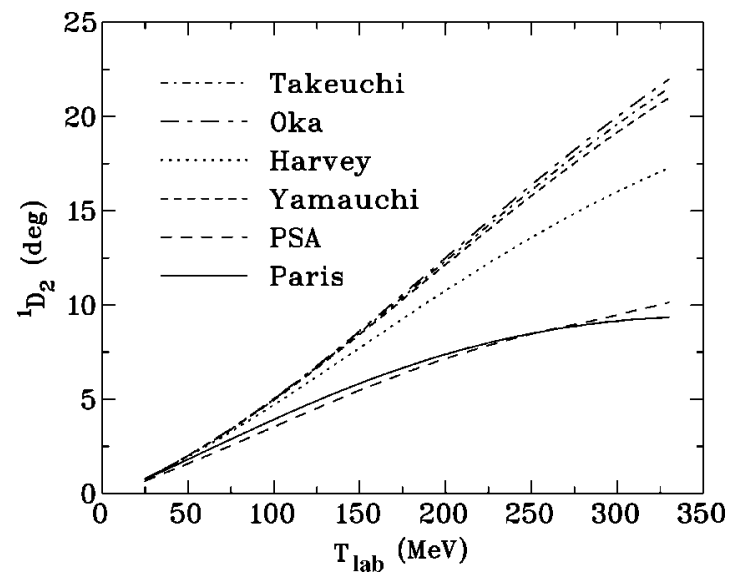

FIG. 5. ${ }^{1} D_{2}$ phase shifts. The curves are labeled as in Fig. 4. 


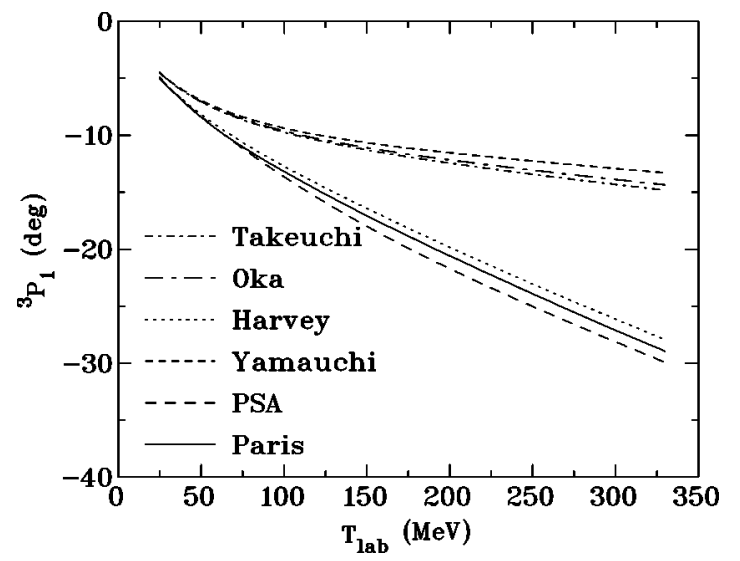

FIG. $6 .{ }^{3} P_{1}$ phase shifts. The curves are labeled as in Fig. 4.

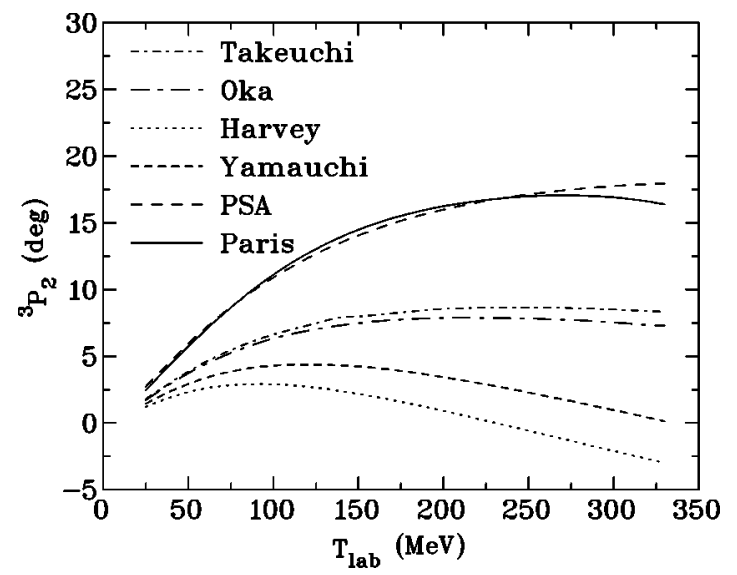

FIG. $7 .{ }^{3} P_{2}$ phase shifts. The curves are labeled as in Fig. 4.

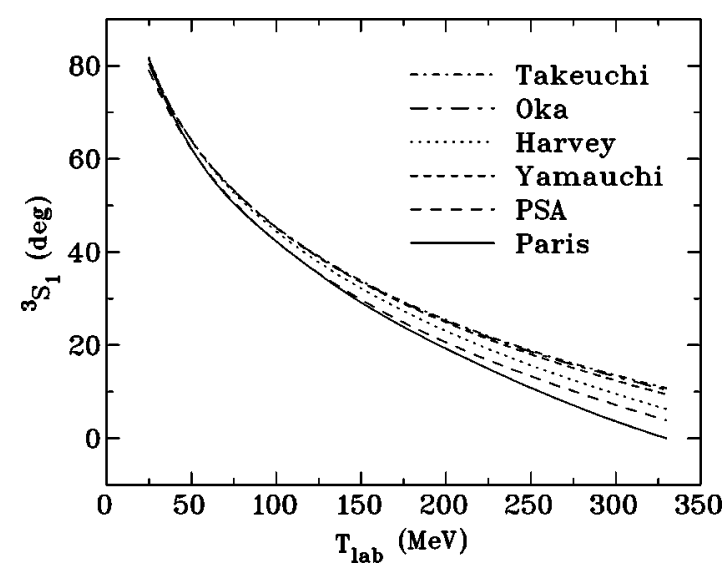

FIG. $8 .{ }^{3} S_{1}$ phases shifts. The curves are labeled as in Fig. 4.

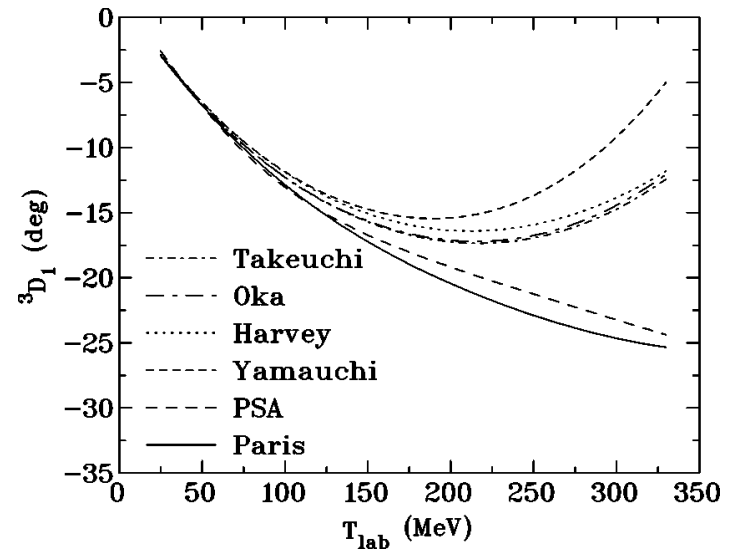

FIG. 9. ${ }^{3} D_{1}$ phase shifts. The curves are labeled as in Fig. 4.

$a_{n}$, the strong coupling constant $\alpha_{s}$, and the oscillator parameter $b_{q}$, they are adjusted to reproduce the baryon properties in the one-baryon sector (three-quark systems). Their values, found in different works $[6,7,11,15]$, are listed in Table I. We have also included the model of quark-quark interaction considered in Ref. [16]

$$
\begin{gathered}
V_{i j}^{c o n f}=\boldsymbol{\lambda}_{i} \cdot \boldsymbol{\lambda}_{j}\left[A \exp \left(-\frac{r_{i j}^{2}}{\Lambda^{2}}\right)+B r_{i j}^{2}+C\right], \\
V_{i j}^{O G E}=K \boldsymbol{\lambda}_{i} \cdot \boldsymbol{\lambda}_{j}\left(1+\frac{2}{3} \boldsymbol{\sigma}_{i} \cdot \boldsymbol{\sigma}_{j}\right) \delta\left(r_{i j}\right) .
\end{gathered}
$$

The values of $A, B, C, K$, and $\Lambda$ are listed in Table II. They were obtained by fitting also to the baryon spectrum.

\section{RESULTS}

\section{A. Calculation of the potential $V_{Q C M}\left(r, r^{\prime}\right)$}

We have built a general numerical code from the formulas of Ref. [15] to generate the $V_{Q C M}\left(\mathbf{r}, \mathbf{r}^{\prime}\right)$ from the different quark-quark interaction models of Refs. [6,7,11,15,16]. In the course of our calculations we found it necessary to correct some numerical factors. A factor 3 is missing in the $T$

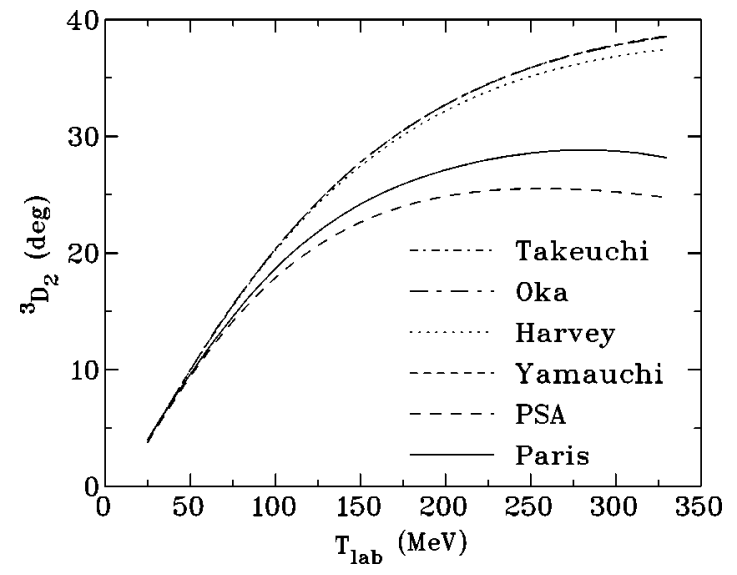

FIG. 10. ${ }^{3} D_{2}$ phase shifts. The curves are labeled as in Fig. 4. 
TABLE IV. The deuteron properties. The different models are as in Table III. $\epsilon$ is the deuteron binding energy. Numbers in square brackets indicate references to source papers.

\begin{tabular}{lccccccc}
\hline \hline $\begin{array}{l}\text { Model with } \\
\text { parameters of }\end{array}$ & $\begin{array}{l}R_{c}(\mathrm{fm}) \\
\text { fitted to }\end{array}$ & $Q\left(\mathrm{fm}^{2}\right)$ & $\epsilon(\mathrm{MeV})$ & $\mu / \mu_{0}$ & $A_{D} / A_{S}$ & $P_{D}(\%)$ & $\sqrt{\left\langle r^{2}\right\rangle}(\mathrm{fm})$ \\
\hline Takeuchi [11] & 0.8059 & 0.283 & -2.226 & 0.844 & 0.0262 & 6.25 & 1.984 \\
Oka [6] & 0.8064 & 0.282 & -2.223 & 0.844 & 0.0261 & 6.18 & 1.986 \\
Yamauchi [15] & 0.7987 & 0.278 & -2.226 & 0.848 & 0.0258 & 5.49 & 1.985 \\
Faessler [7] & 0.8064 & 0.279 & -2.223 & 0.847 & 0.0259 & 5.74 & 1.986 \\
Harvey [16] & 0.9090 & 0.276 & -2.224 & 0.849 & 0.0257 & 5.37 & 1.987 \\
Paris potential [5] & & 0.279 & -2.225 & 0.853 & 0.0261 & 5.77 & 1.950 \\
Experiment & & $0.2859(3)$ & $-2.224575(9)$ & $0.8574(1)$ & $0.0256(4)$ & & $1.971(6)$ \\
& & {$[17]$} & {$[18]$} & {$[19]$} & {$[20]$} & & {$[21]$} \\
\hline \hline
\end{tabular}

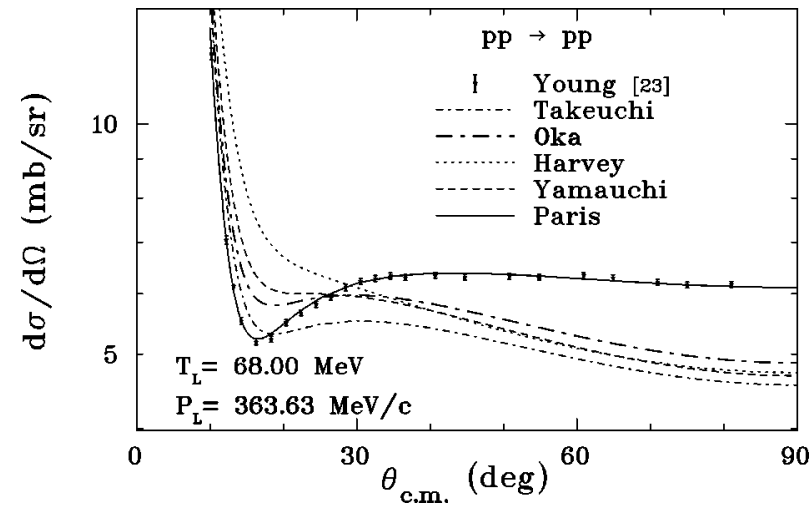

FIG. 11. $p p$ elastic differential cross section at $T_{l a b}=68 \mathrm{MeV}$. The curves correspond to the different QCM, supplemented with the LR+MR part of the Paris potential. Paris refers to results of Ref. [5] and Young refers to experimental results from Ref. [23].

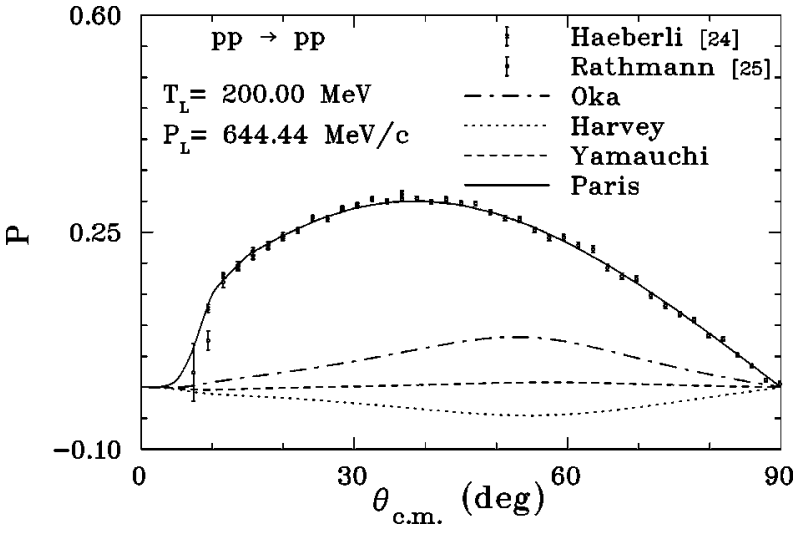

FIG. 12. $p p$ elastic polarization at $T_{l a b}=200 \mathrm{MeV}$. The curves are labeled as in Fig. 11. Rathmann and Haeberli correspond to results from Refs. [25] and [24], respectively.

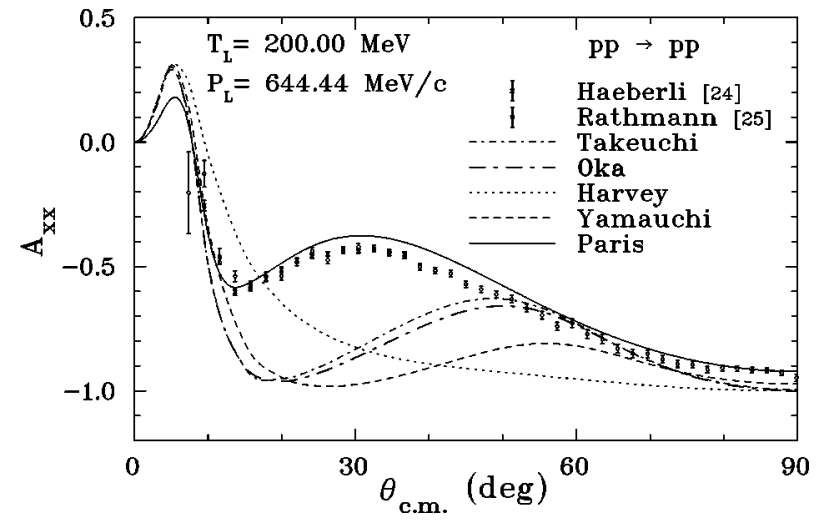

FIG. 13. $p p$ spin correlation parameter $A_{x x}$ at $T_{l a b}=200 \mathrm{MeV}$. The curves are labeled as in Fig. 11.

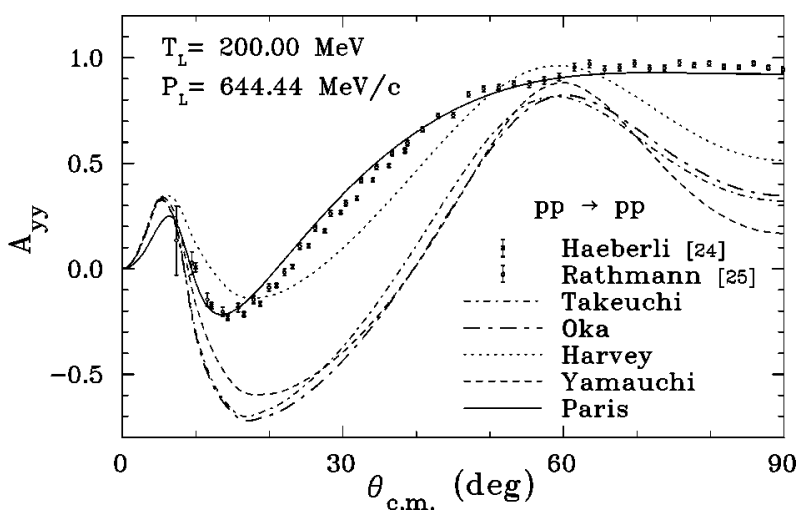

FIG. 14. $p p$ spin correlation parameter $A_{y y}$ at $T_{l a b}=200 \mathrm{MeV}$. The curves are labeled as in Fig. 11. 


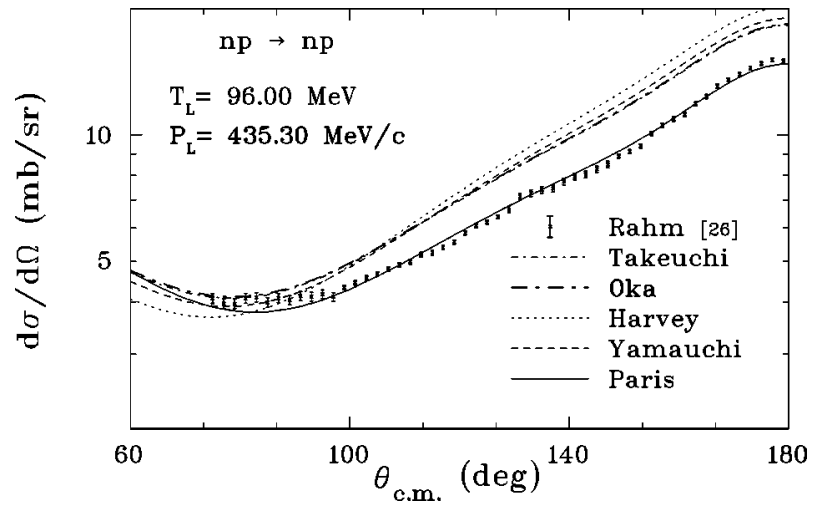

FIG. 15. $n p$ elastic differential cross section at $T_{l a b}=96 \mathrm{MeV}$. The curves are labeled as in Fig. 11. Rahm shows results from Ref. [26].

$=1$ local triplet central potential, a factor $-2 / \sqrt{5}$ in the $T$ $=1$ local tensor, a factor $1 /(2 \sqrt{5})$ in the nonlocal tensor, a factor $\sqrt{6} / 3$ in the nonlocal spin-orbit potentials, a factor 2 in the ${ }^{3} S_{1}-{ }^{3} D_{1}$, and ${ }^{3} P_{1}-{ }^{3} F_{2}$ tensor potentials. We have checked that these modifications enable us to reproduce the numerical results for $V_{Q C M}\left(\mathbf{r}, \mathbf{r}^{\prime}\right)$ of Ref. [11]. Some typical shapes of nonlocal potentials obtained with the parameter set of Ref. [11] are displayed in Figs. 1-3. In these figures, we have plotted, as functions of $r$ and $r^{\prime}$, the partial wave projections of $V_{Q C M}$ multiplied by $r r^{\prime}$.

\section{B. Comparison of the phase shifts}

We have solved Eq. (2.1) using the different QCM potentials $V_{Q C M}$ calculated in the preceding section, together with the LR+MR parts of Paris potential [5]. We did not include the $\omega$-meson exchange. The only remaining free parameters are those of the cutoff function $f(r)$, namely, $p$ and $R_{c}$. To make a clear separation between the short-range QCM potential and the meson-exchange potential, we chose $p=10$. Regarding the cutoff radius $R_{c}$, we determine it, as in Ref. [12], by fitting to the ${ }^{1} S_{0}$ phase shift at $25 \mathrm{MeV}$ in the isospin $T=1$ channel, and to the deuteron binding energy $\epsilon$ in the isospin $T=0$ channel. The best values of $R_{c}$ obtained with the different QCM potentials using different sets of

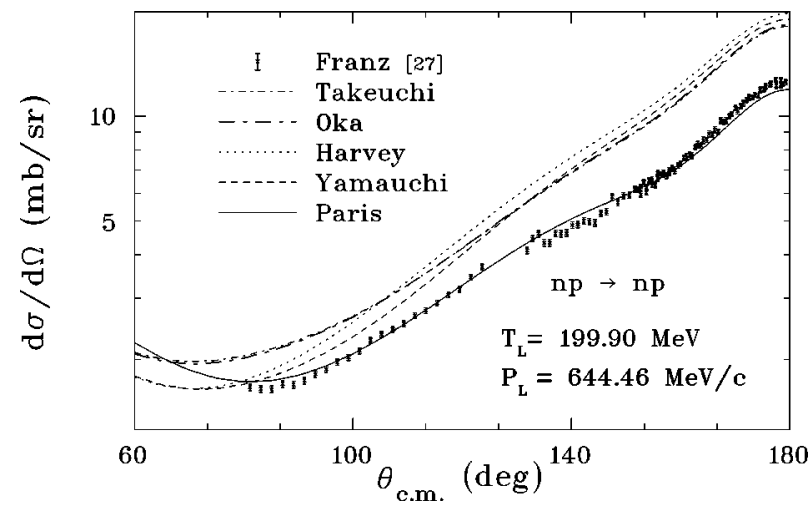

FIG. 16. $n p$ elastic differential cross section at $T_{\text {lab }}$ $=200 \mathrm{MeV}$. The curves are labeled as in Fig. 11. Franz shows results from Ref. [27].

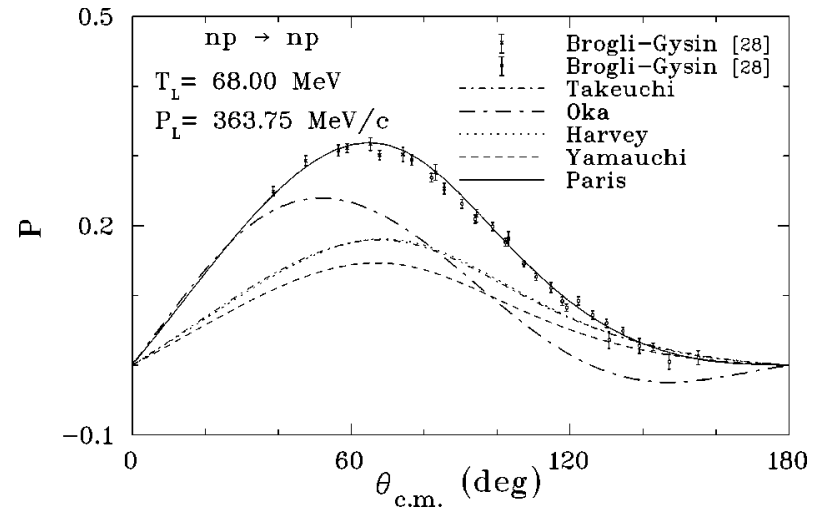

FIG. 17. $n p$ elastic polarization at $T_{l a b}=68.0 \mathrm{MeV}$. The curves are labeled as in Fig. 11. Brogli-Gysin data points are from Ref. [28].

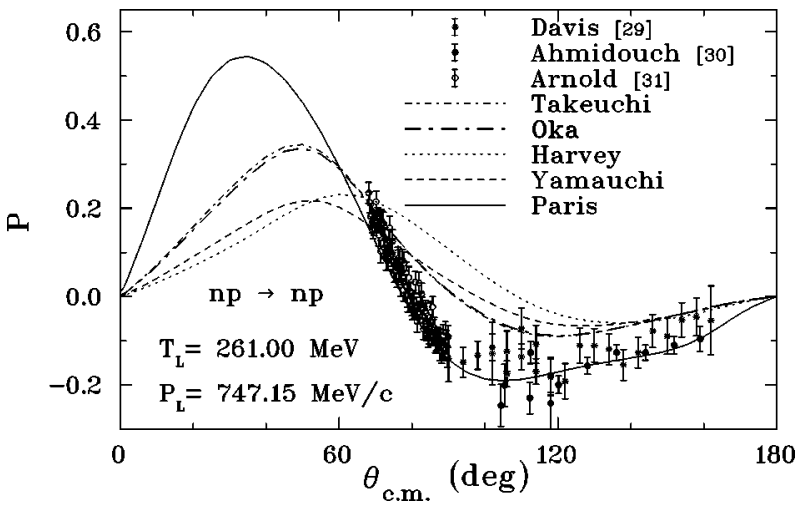

FIG. 18. $n p$ elastic polarization at $T_{l a b}=261 \mathrm{MeV}$. The curves are labeled as in Fig. 11. Davis, Ahmidouch, and Arnold are from results of Refs. [29-31], respectively.

TABLE V. $\chi^{2} /$ data for $p p$ and $n p$ observables. The different models are as in Table III. The fit performed by Takeuchi et al. in Ref. [11], the Paris potential results, and the PSA are also shown.

\begin{tabular}{lcc}
\hline \hline & $p p$ & $n p$ \\
Models & $\begin{array}{c}\text { (1353 data from } \\
\text { 25 to 333 MeV) }\end{array}$ & $\begin{array}{c}\text { (2268 data from } \\
\text { 25 } 325 \mathrm{MeV})\end{array}$ \\
\hline Oka [6] & 140.28 & 20.34 \\
Yamauchi [15] & 230.57 & 36.00 \\
Takeuchi [11] & 143.20 & 19.00 \\
Faessler [7] & 228.06 & 34.00 \\
Harvey [16] & 360.6 & 30.36 \\
Takeuchi [11] (with phase- & & \\
dependent $R_{c}$ of Table VI) & 13.34 & 13.36 \\
Takeuchi [11] & 13.25 & 25.24 \\
(adjustable meson exchanges) & & \\
Paris potential [5] & 1.96 & 2.83 \\
PSA [22] & 1.40 & 1.61 \\
\hline \hline
\end{tabular}


TABLE VI. Best values of the partial-wave dependent cut off radius $R_{c}$ with the model of Takeuchi [11].

\begin{tabular}{lccccccc}
\hline \hline & \multicolumn{3}{c}{ Isospin 1} \\
\hline Phases & ${ }^{1} S_{0}$ & ${ }^{1} D_{2}$ & ${ }^{3} P_{0}$ & ${ }^{3} P_{1}$ & ${ }^{3} P_{2}$ & ${ }^{1} P_{1}$ & ${ }^{3} D_{2}$ \\
$R_{c}$ (fm) (fit of PSA phases) & 0.830 & 1.130 & 0.956 & 1.119 & 0.688 & 0.600 & 1.200 \\
\hline \hline
\end{tabular}

quark-quark parameters are shown in Table III. Interestingly enough they are close to the values $(0.8 \mathrm{fm})$ we adopted for the separation between the theoretical and phenomenological parts of the Paris potential [5].

For a first qualitative comparison we display in Figs. 4-10 the results of some phase shifts obtained from the different QCM of Refs. $[6,7,11,15,16]$ with their corresponding best values for $R_{c}$, as listed in Table III. We restrict ourselves to the low partial waves $(J \leqslant 2)$ since these are the most sensitive to the SR part of the potential. They are compared with the recent phase shift analysis (PSA) [22] as well as with the Paris potential results. The results for the deuteron properties [17-21] corresponding to the same QCM models are shown in Table IV. The ${ }^{1} S_{0}$ phase shift is well reproduced, as $R_{c}$ is adjusted to fit it at $25 \mathrm{MeV}$. However the ${ }^{3} S_{1}$ phase is not so good. The agreement for the other phases is rather poor, especially in the higher-energy regions where one expects the role of the quark degrees of freedom to become more dominant.

\section{Comparison with scattering observables}

We believe that the comparison with phase shifts does not provide a severe enough model testing bench. For a more meaningful test we confront the predictions directly with the data on observables. We have performed such a comparison using the presently available world set of $N N$ scattering data up to $350 \mathrm{MeV}$. All observables have been calculated and some examples are shown in Figs. 11-18 for $p p$ as well as $n p$ scattering. As it can be seen, the agreement with experiment [23-31] is again poor. This is generally true for the other observables as well, leading to the values of the total $\chi^{2} /$ data listed in Table V. The model fails to reproduce not only spin observables but also cross sections. It is worth noticing that the differences in the quark-quark interactions show up more manifestly in the spin observables. One might argue that choosing $R_{c}$ to be dependent only on isospin states is a too drastic prescription. We have tried to leave it free and to carry out a best fit of the partial waves. This leads to values shown in Table VI. It results in a better $\chi^{2} /$ data, shown also in Table $\mathrm{V}$, but without improving really the fit to the observables.

\section{Discussion and concluding remarks}

In this work, a careful and detailed analysis of the short distance effects of quark degrees of freedom on the $N N$ observables was carried out, in the framework of the QCM. The agreement of the theoretical predictions with data is quantitatively poor, leading to conclusions different from those found generally in the literature. Changes in the choice of quark-quark interactions do not improve the situation. It is thus clear that the assessment of the ability of the QCM to describe the SR nucleon-nucleon forces differs, depending on whether one adopts the viewpoint of supplementing the QCM with adjustable LR + MR forces or one chooses to associate the QCM with well-established LR+MR forces. It could seem surprising that the nonrelativistic quark model, which has achieved remarkable successes in describing the static properties of single hadron, fails to reproduce quantitatively the SR part of the $N N$ interaction. It is, of course, easy to blame the approximation made in the QCM, but it is fair to admit that so far no better method has been proposed and put in practice. In this work we focused on the QCM related to the original nonrelativistic quark model with quark and gluon degrees of freedom. The same type of investigations can be contemplated for a quark model with Goldstone boson, instead of gluon, exchanges as residual interaction. Some recent calculations were carried out, in this direction, for the nucleon-nucleon ${ }^{1} S_{0}$ and ${ }^{3} S_{1}$ phase shifts [32]. The results obtained, namely, a strong repulsion, are reminiscent of those in the early works [6-9]. Which of the two approaches is more relevant to QCD remains an open theoretical issue.

\section{ACKNOWLEDGMENTS}

Parts of this work were carried out during different stays of three of us, P.D., J.P.B.C. de M., and C.S., at LPTPE, Université P. et M. Curie, Paris. The same authors acknowledge the financial support of the EU Human Capital and Mobility Program (network ERBCHRXCT 390-323), of the Brazilian Agency FAPESP (Contract No. 97/13902-8), and of the Institut Interuniversitaire des Sciences Nucléaires (Belgium), respectively. Laboratoire de Physique Nucléaire et des Hautes Energies is Unité de Recherche des Universités Paris 6 et Paris 7, associée au CNRS.
[1] J. J. de Swart, R. A. M. M. Klomp, M. C. M. Rentmeester, and Th. A. Rijken, Few-Body Syst., Suppl. 8, 437 (1995).

[2] R. Vinh Mau, in Recent Advances in Hadron Physics, edited by K. Kang et al. (World Scientific, Singapore, 1998), p. 295.

[3] R. Machleidt and I. Slaus, J. Phys. G 27, 69 (2001).

[4] R. Machleidt, K. Holinde, and Ch. Elster, Phys. Rep. 149, 1 (1987).
[5] M. Lacombe, B. Loiseau, J.-M. Richard, R. Vinh Mau, P. Pirès, and R. de Tourreil, Phys. Rev. D 12, 1495 (1975); M. Lacombe, B. Loiseau, J.-M. Richard, R. Vinh Mau, J. Côté, P. Pirès, and R. de Tourreil, Phys. Rev. C 21, 861 (1980).

[6] M. Oka and K. Yazaki, Phys. Lett. 90B, 41 (1980); Prog. Theor. Phys. 66, 556 (1981); Nucl. Phys. A402, 477 (1983).

[7] M. A. Faessler, F. Fernandez, G. Lübeck, and K. Shimizu, 
Phys. Lett. 112B, 201 (1982); Nucl. Phys. A402, 555 (1983).

[8] Y. Suzuki and K. T. Hecht, Phys. Rev. C 28, 1458 (1983); 29, 1586 (1984).

[9] Y. Fujiwara and K. T. Hecht, Nucl. Phys. A444, 541 (1985); A451, 625 (1986); A456, 699 (1986); Phys. Lett. B 171, 17 (1986).

[10] Y. Yamauchi, R. Yamamoto, and M. Wakamatsu, Nucl. Phys. A443, 628 (1985).

[11] S. Takeuchi, K. Shimizu, and K. Yazaki, Nucl. Phys. A504, 777 (1989).

[12] R. Vinh Mau, C. Semay, B. Loiseau, and M. Lacombe, Phys. Rev. Lett. 67, 1392 (1991); C. Semay, Thèse de Doctorat, Université P. et M. Curie, Paris, 1993.

[13] D. Hadjimichef, J. Haidenbauer, and G. Krein, Phys. Rev. C 63, 035204 (2001).

[14] Guanghan Wu, Lijian Teng, J. L. Ping, F. Wang, and T. Goldman, Phys. Rev. C 53, 1161 (1996).

[15] Y. Yamauchi, K. Tsushima, and M. A. Faessler, Few-Body Syst. 12, 69 (1992).

[16] M. Harvey, Nucl. Phys. A352, 326 (1981).

[17] D. M. Bishop and L. M. Cheung, Phys. Rev. A 20, 381 (1979); T. E. O. Ericson and M. Rosa-clot, Nucl. Phys. A405, 497 (1983).

[18] C. van der Leun and C. Alderlisten, Nucl. Phys. A380, 261 (1982).
[19] T. F. Wimmett, Phys. Rev. 91, 499 (1953); T. E. O. Ericson and W. Weise, Pions and Nuclei (Clarendon, Oxford, 1998), p. 50.

[20] N. L. Rodning and L. D. Knutson, Phys. Rev. C 41, 898 (1990).

[21] F. Schmidt-Kaler, D. Leibfried, M. Weitz, and T. W. Hänsch, Phys. Rev. Lett. 70, 2261 (1993); K. Pachucki, M. Weitz, and T. W. Hänsch, Phys. Rev. A 49, 2255 (1994); J. Martorell, D. W. L. Sprung, and D. C. Zheng, Phys. Rev. C 51, 1127 (1995).

[22] R. A. Arndt, W. J. Brisc, R. L. Workman, and I. I. Strakovsky, SAID-SM00, http://gwdac.phys.gwu.edu

[23] D. E. Young and L. H. Johnston, Phys. Rev. 119, 313 (1960).

[24] W. Haeberli et al., Phys. Rev. C 55, 597 (1997).

[25] F. Rathmann et al., Phys. Rev. C 58, 658 (1998).

[26] J. Rahm et al., Phys. Rev. C 63, 044001 (2001).

[27] J. Franz, E. Rösslle, and H. Schmitt, Phys. Scr. T87, 14 (2000).

[28] C. Brogli-Gysin, S. Burzynski, J. Campbell, P. Haffter, M. Hammans, R. Henneck, J. Jourdan, G. Masson, M. A. Pickar, and I. Sick, Nucl. Phys. A541, 137 (1992).

[29] C. A. Davis et al., Phys. Rev. C 53, 2052 (1996).

[30] A. Ahmidouch et al., Eur. Phys. J. C 2, 627 (1998).

[31] J. Arnold B. van den Brandt et al., Eur. Phys. J. C 17, 83 (2000).

[32] D. Bartz and F. L. Stancu, Phys. Rev. C 63, 034001 (2001), and related references cited therein. 